\title{
Finite amplitude flexural vibrations at ultrasonic frequencies in metallic bars
}

\author{
C. Campos-Pozuelo and J. A. Gallego-Juárez \\ Instituto de Acústica, CSIC Serrano, 144, 28006 Madrid, Spain
}

(Received 28 September 1994; revised 1 February 1995; accepted 10 April 1995)

\begin{abstract}
This paper deals with a theoretical and experimental study about the nonlinear behavior of metals subjected to intense ultrasonic, flexural vibrations. In the theoretical analysis a one-dimensional, second-order wave equation is established and solved by means of the successive approximation method. The solution for 'standing, flexural waves is obtained by applying the superposition principle. Spatial distributions of force, momentum, and particle velocity are derived as well as the waveforms. The experimental study is carried out with resonant, prismatic bars driven at their central sections at frequencies in the range of $20-30 \mathrm{kHz}$. The samples are driven by means of a piezoelectric transducer. The vibration amplitudes and waveforms are monitored by using a laser vibrometer. Good agreement is found comparing the experimental and theoretical results. (C) 1995 Acoustical Society of America.
\end{abstract}

PACS numbers: $43.25 . \mathrm{Vt}, 43.35 . \mathrm{Yb}, 43.40 . \mathrm{Cw}$

\section{INTRODUCTION}

It is well known that, only in the case of infinitesimal amplitudes, the vibration of elastic solids can be described by linear laws. In fact, if the vibration is of finite amplitude, the strain and stress tensors contain higher-order terms and they are no longer linearly related. ${ }^{1,2}$ As a consequence, the equations of motion become nonlinear. ${ }^{3,4}$ Therefore, vibrations in high-power transducers, which are of finite amplitude have to be studied in the nonlinear range. Two types of waves are commonly applied in these transducers: Extensional and flexural waves. ${ }^{5}$

In the literature there exists a number of theoretical and experimental investigations about the behavior of nonlinear, pure longitudinal, ${ }^{3,4,6-8}$ and transverse ${ }^{9}$ progressive waves. But few analyses are found about extensional and flexural waves. In addition, finite amplitude standing waves have been scarcely studied and the majority of the published works refer to fluids. ${ }^{10,11}$ Nonlinearly vibrating, elastic solids in resonance have only recently found the attention of some investigators $^{12}$ considering longitudinal finite amplitude waves. Extensional standing waves, being the most common ones in high-power ultrasonic transducers, were studied previously by the authors of this paper. ${ }^{13}$ No information is found about flexural standing waves.

In this paper, a theoretical and experimental study of finite-amplitude, flexural standing waves in resonant bars of perfectly elastic, isotropic solids is presented. The theoretical analysis establishes a one-dimensional, second-order flexural wave equation which is solved by means of the successive approximation method. ${ }^{14}$ The solution for standing flexural waves is obtained by applying the superposition principle. The experimental study is carried out with resonant, prismatic bars driven at their central sections at frequencies in the range of $20-30 \mathrm{kHz}$. The samples employed in this study are made of titanium alloy (Ti $6 \mathrm{Al} 4 \mathrm{~V}$ ) which is commonly used in the construction of high-power ultrasonic transducers. $^{5}$

\section{THEOAY}

This study considers flexural waves in an isotropic, prismatic bar with small $d / \lambda$ and $h / \lambda$ ratio (Fig. 1) ( $d$ and $h$ being the two transversal dimensions of the bar and $\lambda$ the wavelength in the material). Under these conditions it is assumed that the vibration of the specimen can be described by a one-dimensional model. In the present approach losses are neglected. This assumption can be justified by taking into account that the studied solids are polycrystalline metals in which the dissipation energy is small. Only second-order terms of the strain are considered.

\section{A. Second-order, one-dimensional wave equation}

The finite amplitude, flexural wave model is based on the nonlinear elasticity theory. All equations in this paper are expressed in Lagrangian coordinates. We call $a$ the coordinate along the axis of the bar (Fig. 1). The temperature of the system is considered to be constant and, therefore, all the elastic constants involved correspond to constant temperature. We consider an isotropic material in which only onedimensional flexural waves are propagating so that the displacement is-normal to the propagation direction and the strain is produced only in the direction of propagation. The well-known one-dimensional, linear, flexural wave equation is $^{15}$

$$
\frac{\partial^{4} u}{\partial a^{4}}=-\frac{\rho_{0}}{Y_{0} \kappa^{2}} \frac{\partial^{2} u}{\partial t^{2}} .
$$

The momentum and the shear force are given by ${ }^{15}$

$$
\begin{aligned}
& m=-S Y_{0} \kappa^{2} \frac{\partial^{2} u}{\partial a^{2}}, \\
& f=\frac{\partial m}{\partial a}=-S Y_{0} \kappa^{2} \frac{\partial^{3} u}{\partial a^{3}},
\end{aligned}
$$

where $u$ is the displacement, $\rho_{0}$ the density at the initial state, $Y_{0}$ the Young modulus, $S$ the cross-sectional surface area of 


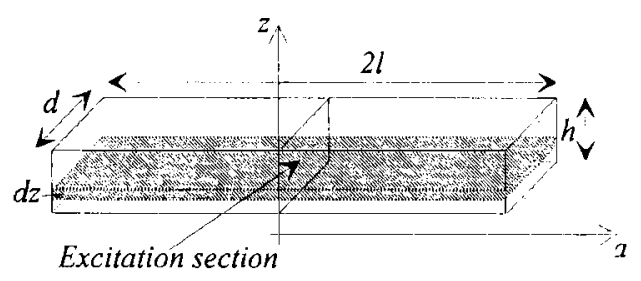

FIG. 1. Scheme of a prismatic bar.

the bar, and $\kappa$ the radius of gyration. For a parallelepiped bar $\kappa=h / \sqrt{12}$.

The linear, extensional strain for every longitudinal element $d z$ (Fig. 1) results to be,

$$
\eta=-\kappa^{2} \frac{\partial^{3} u}{\partial a^{3}}
$$

In a second-order approximation, the stress expuessed in Lagrangian coordinates (the Piola-Kirchoff tensor) is $P=Y_{0} \eta+Y_{1} \eta^{2}{ }^{1}$ Taking into account (4) it results

$$
P=-Y_{0} \kappa^{2} \frac{\partial^{3} u}{\partial a^{3}}+Y_{1}\left(\kappa^{2} \frac{\partial^{3} u}{\partial a^{3}}\right)^{2} \text {, }
$$

where

$$
Y_{0}=\frac{\mu^{*}\left(3 \lambda^{*}+2 \mu^{*}\right)}{\lambda^{*}+\mu^{*}}
$$

and

$$
\begin{aligned}
Y_{1}= & \left(3 \frac{\mu^{*}\left(3 \lambda^{*}+2 \mu^{*}\right)}{\lambda^{*}+\mu^{*}}+\frac{\mu^{* 3}}{\left(\lambda^{*}+\mu^{*}\right)^{3}} l\right. \\
& \left.+\frac{\mu^{*}\left(3 \lambda^{*}+2 \mu^{*}\right)^{2}}{2\left(\lambda^{*}+\mu^{*}\right)^{3}} m+\frac{3 \lambda^{* 2}}{4\left(\lambda^{*}+\mu^{*}\right)^{2}} n\right) / 2
\end{aligned}
$$

being $\lambda^{*}$ and $\mu^{*}$ the second-order elastic moduli (Jamé coefficients) and $l, m$, and $n$ the third-order elastic moduli (Murnaghan coefficients). ${ }^{13}$ The momentum in second-order approximation is

$$
m=S \int P d a
$$

The conservation of the linear momentum law leads to the nonlinear wave equation:

$$
\rho_{0} \frac{\partial^{2} u}{\partial t^{2}}=-Y_{0} \kappa^{2} \frac{\partial^{4} u}{\partial a^{4}}+Y_{1} \kappa^{4} \frac{\partial}{\partial a}\left(\frac{\partial^{3} u}{\partial a^{3}}\right)^{2}
$$

The nonlinear behavior of the material is usually described by the nonlinearity parameter which can be defined as the ratio of the coefficient of the nonlinear term to the linear one in the wave equation (7). ${ }^{16}$ The nonlinearity parameter value depends on the wave-type considered. From the onedimensional approach it is shown that the nonlinearity parameter for flexural waves is $\beta_{F}=\kappa^{2}\left(Y_{1} / Y_{0}\right)=\kappa^{2} \beta_{E}$, being $\beta_{E}$ the nonlinearity parameter for extensional waves. If $\beta_{E}$ is known for the studied material, Eq. (7) is perfectly defined. Note that the nonlinearity parameter for flexural waves depends on the geometry of the bar. To solve Eq. (7) the successive approximation method is applied. ${ }^{14}$ This method as- sumes a solution based on an addition of two terms in the form $u=u_{l}+u_{n l}$, where $u_{l}$ represents the first-order approximation and $u_{n l}$ the second-order approximation. Substituting $u$ in Eq. (7) we obtain

$$
\rho_{0} \frac{\partial^{2} u_{l}}{\partial t^{2}}+Y_{0} \kappa^{2} \frac{\partial^{4} u_{l}}{\partial a^{4}}=0
$$

for the first-order approximation, and

$$
\rho_{0} \frac{\partial^{2} u}{\partial t^{2}}+Y_{0} \kappa^{2} \frac{\partial^{4} u}{\partial a^{4}}=Y_{1} \kappa^{4} \frac{\partial}{\partial a}\left(\frac{\partial^{3} u_{l}}{\partial a^{3}}\right)^{2}
$$

for the second-order approximation.

It was assumed that $\left[\kappa^{2}\left(\partial^{3} u / \partial a^{3}\right)\right]^{2} \cong\left[\kappa^{2}\left(\partial^{3} u_{l} / \partial a^{3}\right)\right]^{2}$, i.e., all the terms of third or higher-order in the linear strain expression were neglected.

For a time harmonic wave, i.e., $u_{l}=e^{j \omega t} U(a)$, the general solution of Eq. (8) is ${ }^{15}$

$$
u_{l}=e^{j \omega t}\left(A_{l} e^{-j \mu a}+B_{l} e^{-\mu a}+C_{l} e^{j \mu a}+D_{l} e^{\mu a}\right),
$$

with $A_{l}, B_{l}, C_{l}$, and $D_{l}$ being constants, $\omega$ being the angular frequency, and $\mu=\sqrt[4]{\rho \omega^{2} / Y_{0} \kappa^{2}}$. If only a progressive wave is considered, traveling in the forward $a$ direction, Eq. (10) is reduced to

$$
u_{l}=e^{j \omega \prime}\left(A_{l} e^{-j \mu a}+B_{l} e^{-\mu a}\right) .
$$

Note that the second term in Eq. (11) decays exponentially with the propagating distance. For example, after a propagating distance of only $\lambda / 10$ it is already reduced by $90 \%$. Therefore, for a progressive wave this term is only significant close to the source. Nevertheless, we are interested in the construction of a compound wave where terms of the type $e^{\mu a}$ might appear [see Eq. (10)] and the dimensions are of the order of $\lambda / 5$. For this reason these terms cannot be ignored in this study.

From Eqs. (11) and (4) the linear strain in the bar can be easily obtained. In Fig. 2 the linear strain and the velocity distribution of a bar which is flexurally excited at its central section is compared with the linear strain distribution of an extensionally vibrating rod excited at one end with the same amplitude and frequency. It can be observed that the maximum strain amplitude is about five times lower for flexural vibration than for extensional vibration while the maximum velocity amplitude for the same conditions is 1.6 larger for the flexural vibration. This linear, theoretical result is very important for practical applications. In fact, it indicates that flexural vibrations are very appropriate for high-power ultrasonic applications where large displacements of the constituent materials of the transducers are required, keeping the strain small to avoid fatigue problems.

Substituting Eq. (11) in Eq. (9), we obtain an inhomogeneous, linear equation which can be solved by classical differential equation methods. The solution results are

$$
\begin{aligned}
u_{n l}= & \left(C e^{-j \sqrt{2} \mu a}+D e^{-\sqrt{2} \mu a}+d e^{-2 \mu a}+b e^{-j 2 \mu a}\right. \\
& \left.+c e^{-(1+j) \mu a}\right) e^{j 2 \omega t},
\end{aligned}
$$

where $d=-p_{F}\left(B_{l}^{2} / 6\right), \quad b=j p_{F}\left(A_{l}^{2} / 6\right), \quad c=-p_{F}\left\lfloor A_{l} B_{l} l\right.$ $8(j-1)], p_{F}=\beta_{F} \mu^{3}$, and $C$ and $D$ are constants depending on the initial conditions. 


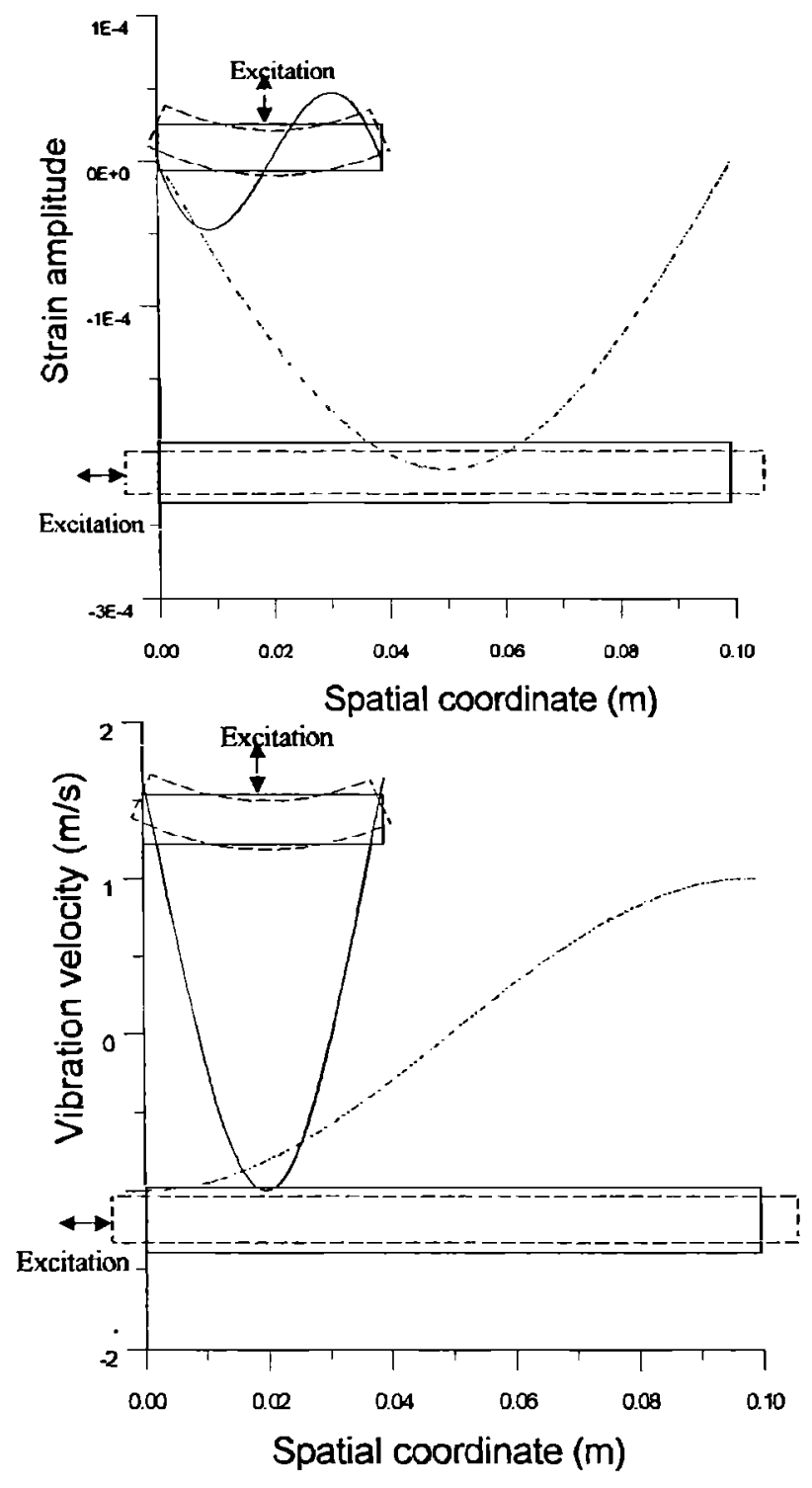

FIG. 2. Theoretical strain and vibration velocity distribution in a prismatic bar vibrating at its first flexural mode (__ first extensional mode (-............) excited with the same amplitude and frequency.

As in the case of linear progressive flexural waves, there are some terms in Eq. (12) which decay exponentially with the propagating distance. Again, for large propagation distances these terms can be neglected while for the construction of the standing waves they are significant.

For sinusoidal excitation, the initial conditions are

$$
u(a=0, t)=u_{0}(t)=u_{0} e^{j \omega t}, \quad f(a=0, t)=0 .
$$

By introducing these conditions in Eqs. (11) and (12) the solution takes the form:

$$
\begin{aligned}
u(a, t)= & \frac{u_{0}(t)}{1+j}\left(e^{-j \mu a}+j e^{-\mu a}\right)+C e^{-j \sqrt{2} \mu a} \\
& +D e^{-\sqrt{2} \mu a}-p_{F} \frac{u_{0}^{2}(t)}{12}\left(\frac{3}{4(j-1)}\right. \\
& \left.\times e^{-(1+j) \mu a}+e^{-2 \mu a}-e^{-j 2 \mu a}\right)
\end{aligned}
$$

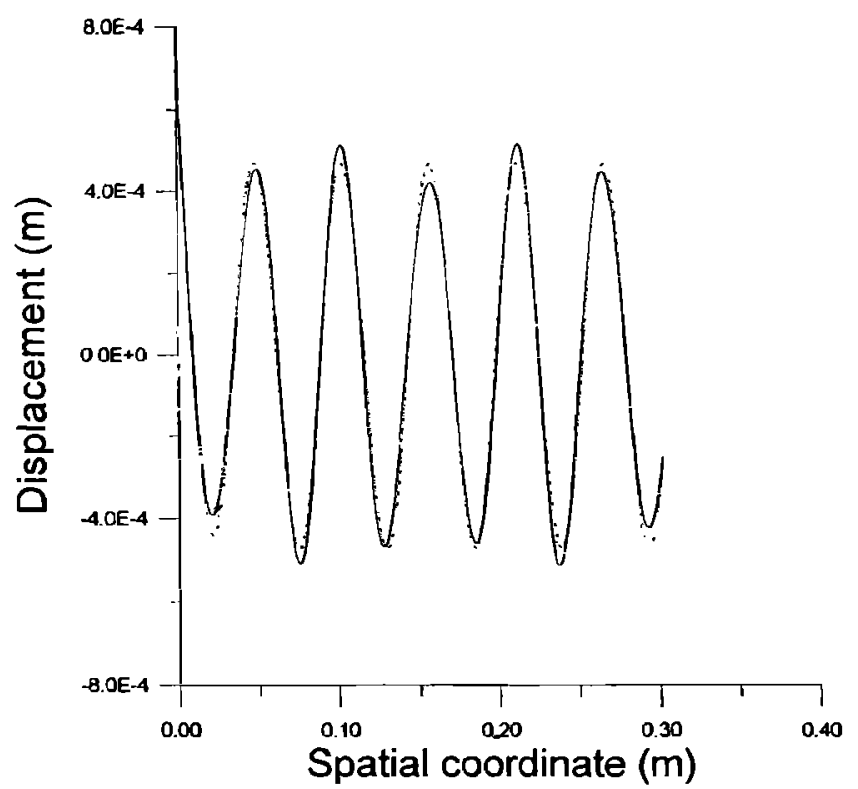

FIG. 3. Theoretical displacement distribution in a semi-infinite bar vibrating flexurally. Linear (-............). Second-order approximation (- - ). Initial displacement, $u_{0}=665 \mu \mathrm{m}$. Frequency, $f=23900 \mathrm{~Hz}$.

being

$C=\frac{d(2 \sqrt{2}-1)-j b(2 \sqrt{2}-j)-c[(1-j+\sqrt{2}) / \sqrt{2}]}{(1+j)}$,

$D=\frac{-d(2 \sqrt{2}+j)+j b(2 \sqrt{2}-1)+c[(1-j-j \sqrt{2}) / \sqrt{2}]}{(1+j)}$

The coefficient $p_{F}$ and the initial amplitude determine the amplitude of the second-order component. This coefficient depends on the nonlinearity parameter, the frequency of the wave, and the geometry of the bar. Note the differences of this solution compared to the known solution for nonlinear longitudinal, progressive waves: ${ }^{4}$ First, the dependence of the flexural solution on the particular geometry of the bar by means of the parameter $\kappa$, second, the presence of spatial amplitude terms of the type $e^{-j \sqrt{2} \mu a}$, i.e., for flexural waves the spatial modulation contains not only terms with twice of the wave number coefficients (one-half of the wavelength) but also with $\sqrt{2}$ coefficients. Moreover, the amplitude of the second-order component does not increase linearly with distance to the sinusoidal source. In Fig. 3, the solution of Eq. (14) is represented and compared to the linear solution. Note the small amplitude of the second-order component for the very high displacements considered. This result is in agreement with the linear results presented in Fig. 2.

\section{B. Nonlinear, flexural standing waves}

The main objective of this work is the study of nonlinear, flexural vibrations in resonant systems such as finite bars. To approach this problem, we consider a onedimensional flexural wave traveling along the axis of the bar, incident on the boundary where it is reflected in opposite 


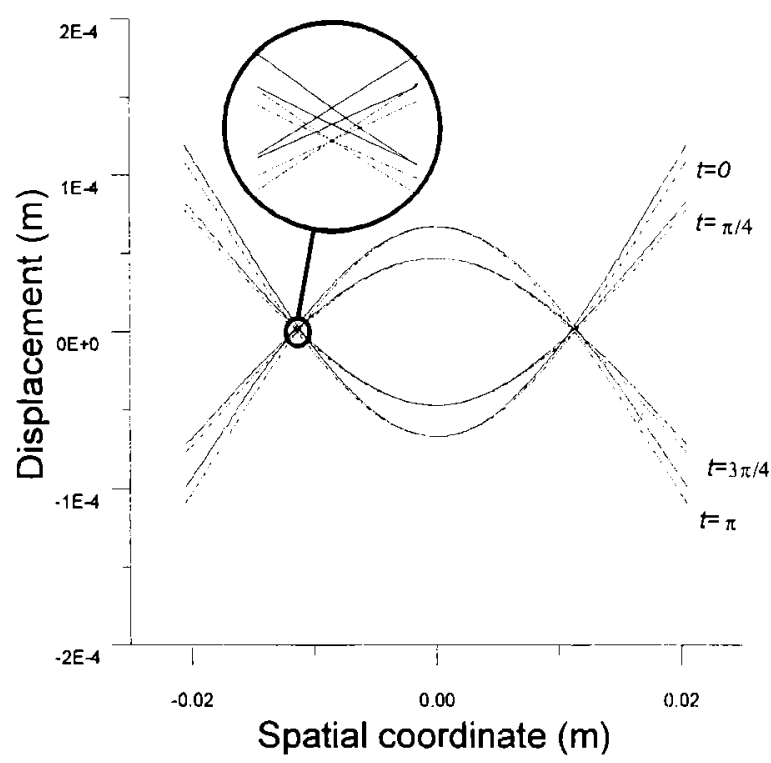

FIG. 4. Theoretical distribution of displacements of a constan section bar vibrating at its first flexural mode at four different times with in a period. Linear $(-. . . . . . .-. .-$.$) . Second-order approximation (- (-)$. Dr ving amplitude $\dot{u}_{0}=10 \mathrm{~m} / \mathrm{s}$. Maximum amplitude $\dot{u}_{\max }=16.5 \mathrm{~m} / \mathrm{s}$. Nonlinearity parameter $\beta_{E}=50$.

direction. To set up a standing wave pattern we assume the superposition of two waves of finite amplitude traveling in forward and backward direction, ${ }^{13}$ i.e.,

$$
u=u_{a}+u_{b} .
$$

The expressions for the linear and nonlinear components of the forward wave $\left(u_{i a}\right.$ and $\left.u_{n l a}\right)$ are shown in Eqs. (11) and (12), respectively, while the expression for the backward wave takes the form:

$$
\begin{aligned}
u_{h}(t, a)= & e^{j \omega t}\left(A_{2} e^{j \mu a}+B_{2} e^{\mu a}\right)+e^{j 2 \omega t}\left(C_{2} e^{j \sqrt{2} \mu a}\right. \\
& \left.+D_{2} e^{\sqrt{2} \mu a}+d_{2} e^{2 \mu a}+b_{2} e^{j 2 \mu a}+c_{2} e^{(1+j) \mu a}\right) .
\end{aligned}
$$

To apply the boundary conditions, the specific system has to be defined. The case studied here is a bar with free ends driven at the central section (Fig. 1). For this geometry and excitation, symmetry is given with respect to the central plane and, thus, it is sufficient to consider only one-half of the bar.

The boundary conditions are

$$
\begin{aligned}
& \left.u(a=0, t)=u_{0}=U_{0} e^{j \omega t}, \quad \frac{\partial u}{\partial a}\right]_{a=0}=0, \\
& f(a=l, t)=0, \quad m(a=l, t)=0, \\
& f(a=0, t)=0 . \quad \text { (resonance condition) }
\end{aligned}
$$

Applying the boundary conditions and neglecting again all third and higher-order terms of $\kappa^{2}\left(\partial^{3} u / \partial a^{3}\right)$, the values of the constants are obtained as a function of $u_{0}$. Applying this result to the particular case of a prismatic resonant bar of constant cross section at $23 \mathrm{kHz}$ and assuming the nonlinearity parameter $\beta_{E}=50$ we obtain for the displacement, force, and momentum the curves represented in Figs. 4 and 5. Note that the conditions for linear and second-order resonance are different. In Fig. 4, the distribution of the displacement is shown at four different times within one period. It is observed that the harmonic generation increases with growing distance from the sinusoidal source. It is also found that, while in the linear approximation the nodes are perfectly defined, in the second-order approximation the locations with zero displacement amplitude change with time, i.e., they are not real nodes. This result follows from the fact that the amplitude of the second harmonic is not null at the node of the fundamental. For the same bar under the same conditions, Fig. 5 shows the force and momentum for the linear and the second-order approximations. It is apparent that the

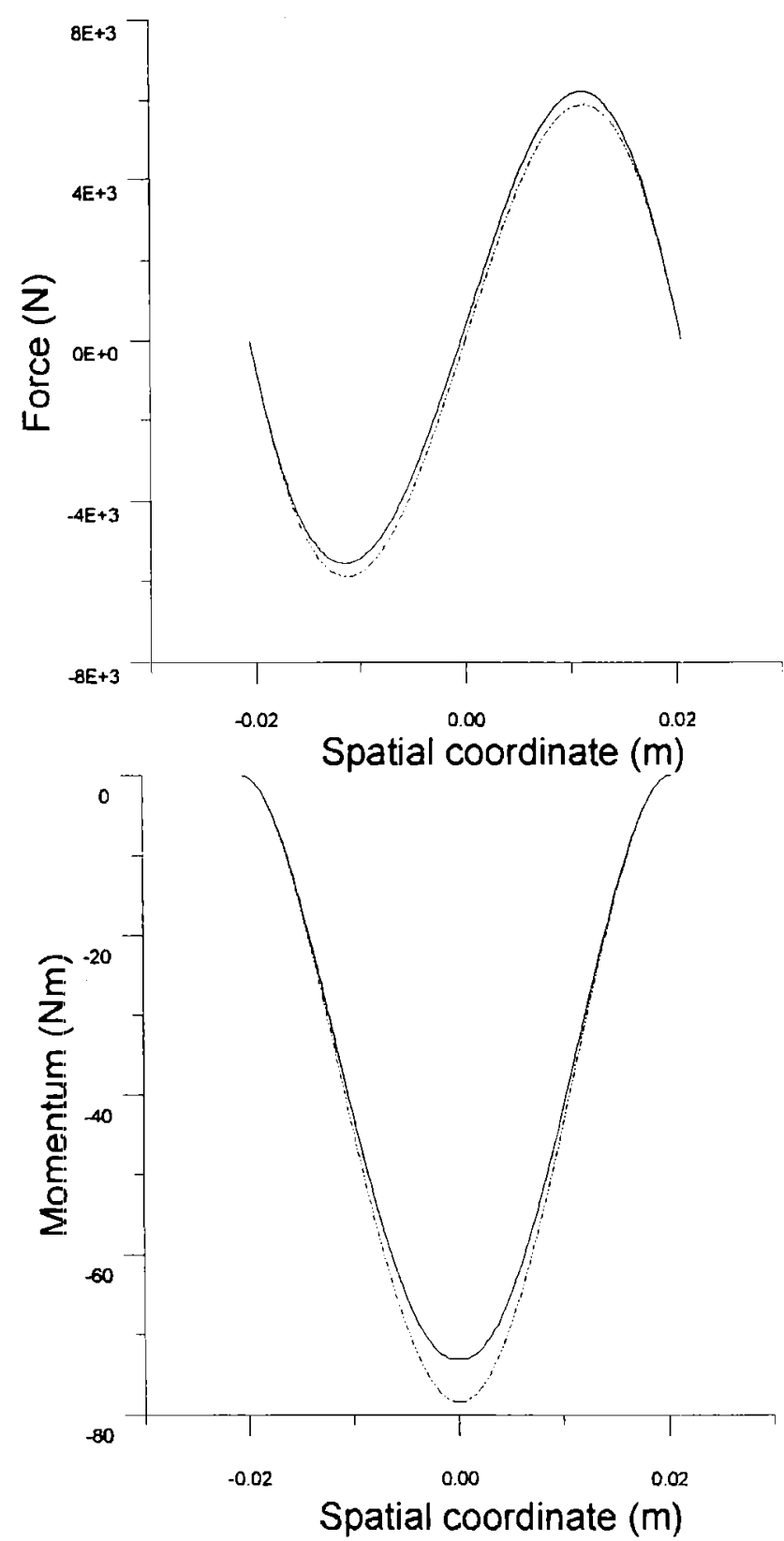

FIG. 5. Theoretical distribution of force and momentum in a constant section bar vibrating at its first flexural mode. Linear (-............). Second-order approximation (—). Driving amplitude $\dot{u}_{0}=10 \mathrm{~m} / \mathrm{s}$. Maximum amplitude $\dot{a}_{\text {max }}=16.5 \mathrm{~m} / \mathrm{s}$. Nonlinearity parameter $\beta_{K}=50$. 


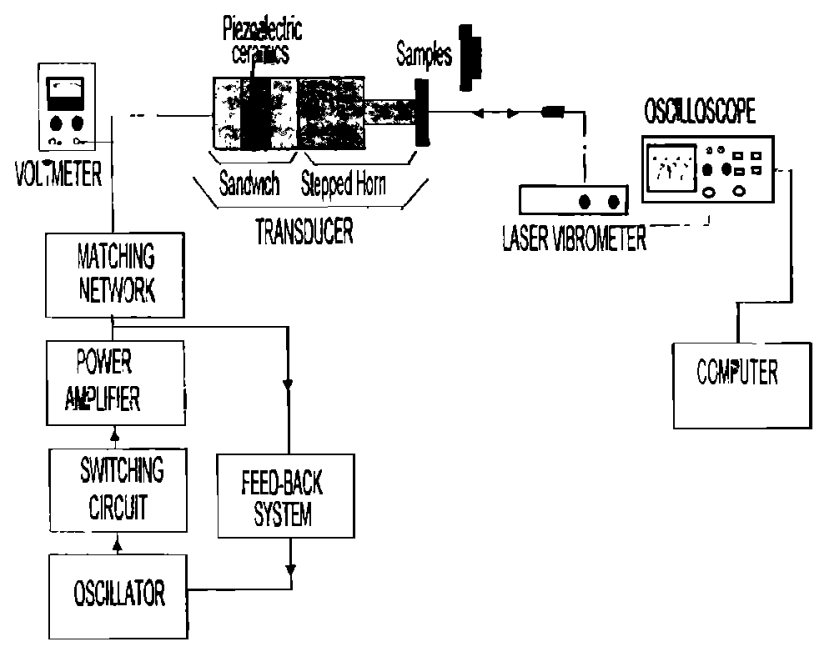

FIG. 6. Experimental setup.

largest differences occur at the center of the bar where the momentum is maximum. Nevertheless, the calculations make clear that the harmonic generation is generally low even if the displacement amplitudes are very high (vibration velocity amplitudes up to $16.5 \mathrm{~m} / \mathrm{s}$ ). This result is expected because of the previously shown low strain amplitudes produced by flexural vibrations.

\section{EXPERIMENTS}

The experimental setup for measuring the amplitude and frequency components of the particle velocity at different points along resonant specimens is shown in Fig. 6. It consists of a driving system to excite the samples at resonance and a data acquisition equipment.

\section{A. Excitation system}

The excitation system consists of a specially designed electronic generator and a piezoelectric transducer. The electronic generator used to drive the transducer implements a feedback system to automatically adjust the excitation frequency to the transducer's resonant frequency. ${ }^{17}$ In addition, a newly designed switching circuit produces periodic interruptions of the excitation signal; this controls the time of excitation to keep the temperature of the sample constant. ${ }^{18}$ The driving transducer, a resonant system at about $23 \mathrm{kHz}$, is constructed from an assembly of two half-wave elements: A piezoelectric sandwich and a stepped horn (see Fig. 6). The sandwich element consists of four piezoelectric ceramics between two metallic cylindrical rods. The stepped horn acts as a mechanical amplifier to achieve higher vibration amplitudes at its thinner termination where the sample is attached.

\section{B. Samples}

The samples, made of a titanium alloy (Ti $6 \mathrm{Al} 4 \mathrm{~V}$, a material commonly used in the construction of high-power transducers), are designed as prismatic bars with uniform and stepped profiles (Fig. 6) and are sized to adjust to the transducer resonance frequency. The samples with stepped profile, designed and constructed to obtain higher strains at the
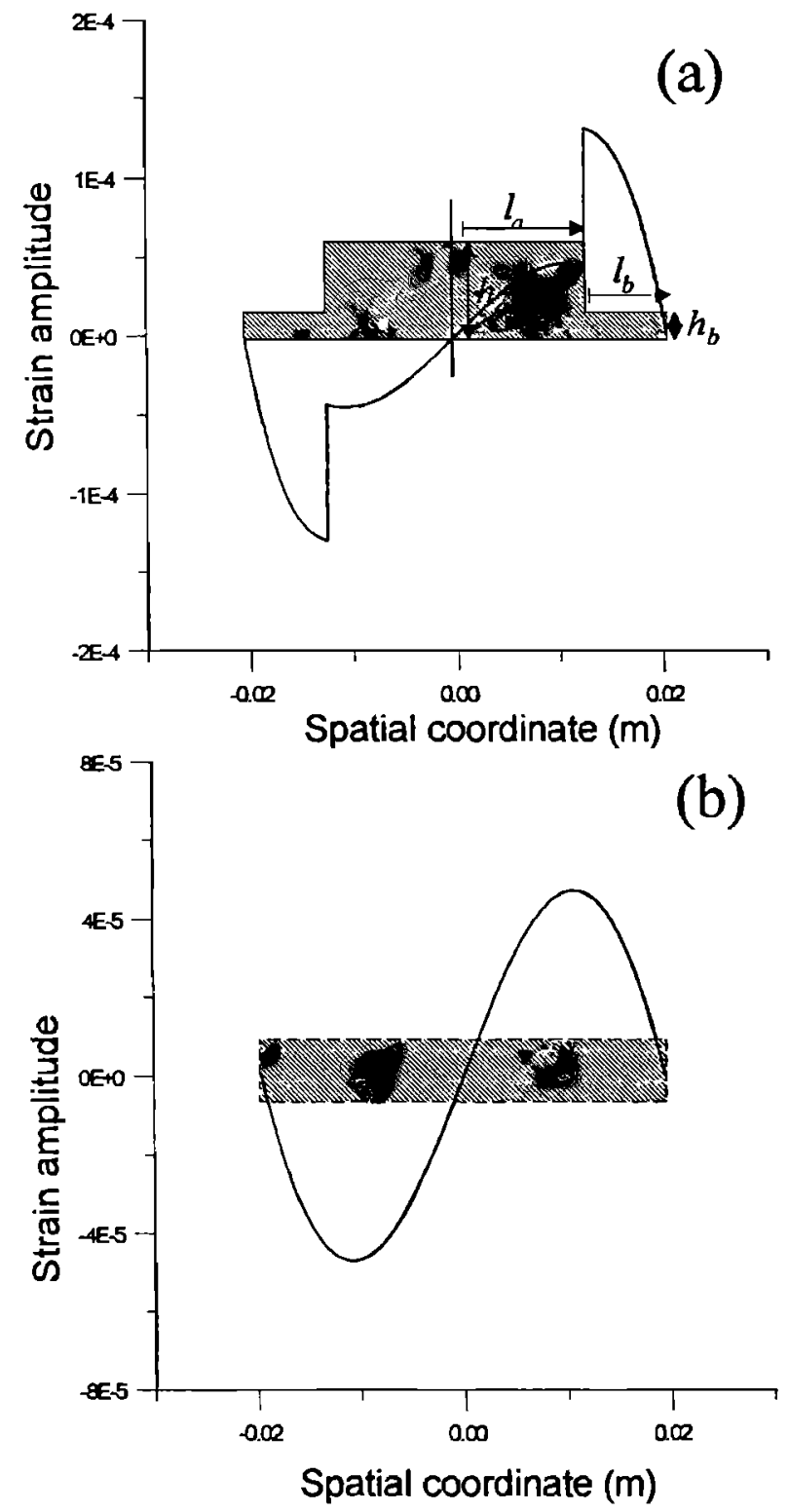

FIG. 7. Theoretical strain distribution in a stepped bar, $h_{a}=8 \mathrm{~mm}, h_{b}=2.5$ $\mathrm{mm}, l_{a}=12.5 \mathrm{~mm}, l_{b}=7.5 \mathrm{~mm}(\mathrm{a})$ and in a uniform section bar (b).

end sections (Fig. 7), lead to higher harmonic generation. For the stepped samples, the resonance condition is obtained from the boundary conditions [Fig. 7(a)] and the continuity conditions (displacement, slope, force, and momentum) in every change of section, ${ }^{19}$ i.e.,

$$
\begin{aligned}
& f\left(a=\left(l_{a}+l_{b}\right)\right)=0, \\
& m\left(a=\left(l_{a}+l_{b}\right)\right)=0, \\
& f\left(a=-\left(l_{a}+l_{b}\right)\right)=0, \\
& m\left(a=-\left(l_{a}+l_{b}\right)\right)=0 .
\end{aligned}
$$

From this one obtains the frequency relation which can be easily solved numerically 
$\frac{E_{a a} \sinh \mu_{b} l_{b}+E_{b a} \cosh \mu_{b} l_{b}+E_{c a} \sin \mu_{b} l_{b}-E_{d a} \cos \mu_{b} l_{b}}{E_{a c} \sinh \mu_{b} l_{b}+E_{b c} \cosh \mu_{b} l_{b}+E_{c c} \sin \mu_{b} l_{b}-E_{d c} \cos \mu_{b} l_{b}}$

$$
=-\frac{E_{a a} \cosh \mu_{b} l_{b}+E_{b a} \sinh \mu_{b} l_{b}-E_{c a} \cos \mu_{b} l_{b}-E_{d a} \sin \mu_{b} l_{b}}{E_{a c} \cosh \mu_{b} l_{b}+E_{b c} \sinh \mu_{b} l_{b}-E_{c c} \cos \mu_{b} l_{b}-E_{d c} \sin \mu_{b} l_{b}},
$$

where

$$
\begin{aligned}
& E_{a a}=\left(1+\frac{S_{a} \kappa_{a}^{2} \mu_{a}^{2}}{S_{b} \kappa_{b}^{2} \mu_{b}^{2}}\right) \cosh \mu_{a} l_{a} / 2, \\
& E_{a c}=\left(1-\frac{S_{a} \kappa_{a}^{2} \mu_{a}^{2}}{S_{b} \kappa_{b}^{2} \mu_{b}^{2}}\right) \cos \mu_{a} l_{a} / 2, \\
& E_{c a}=\left(1-\frac{S_{a} \kappa_{a}^{2} \mu_{a}^{2}}{S_{b} \kappa_{b}^{2} \mu_{b}^{2}}\right) \cosh \mu_{a} l_{a} / 2, \\
& E_{c c}=\left(1+\frac{S_{a} \kappa_{a}^{2} \mu_{a}^{2}}{S_{b} \kappa_{b}^{2} \mu_{b}^{2}}\right) \cos \mu_{a} l_{a} / 2, \\
& E_{b a}=\left(\frac{\mu_{a}}{\mu_{b}}\right)\left(1+\frac{S_{a} \kappa_{a}^{2} \mu_{a}^{2}}{S_{b} \kappa_{b}^{2} \mu_{b}^{2}}\right) \sinh \mu_{a} l_{a} / 2, \\
& E_{b c}=\left(\frac{\mu_{a}}{\mu_{b}}\right)\left(\frac{S_{a} \kappa_{a}^{2} \mu_{a}^{2}}{S_{b} \kappa_{b}^{2} \mu_{b}^{2}}-1\right) \sin \mu_{a} l_{a} / 2, \\
& E_{d a}=\left(\frac{\mu_{a}}{\mu_{b}}\right)\left(1-\frac{S_{a} \kappa_{a}^{2} \mu_{a}^{2}}{S_{b} \kappa_{b}^{2} \mu_{b}^{2}}\right) \sinh \mu_{a} l_{a} / 2, \\
& E_{d c}=-\left(\frac{\mu_{a}}{\mu_{b}}\right)\left(1+\frac{S_{a} \kappa_{a}^{2} \mu_{a}^{2}}{S_{b} \kappa_{b}^{2} \mu_{b}^{2}}\right) \sin \mu_{a} l_{a} / 2, \\
& \mu_{a}=\sqrt[4]{\frac{\rho \omega^{2}}{Y_{0} \kappa_{a}^{2}}} \text { and } \mu_{b}=\sqrt[4]{\frac{\rho \omega^{2}}{Y_{0} \kappa_{b}^{2}}} .
\end{aligned}
$$

The effect of the section discontinuity on the strain amplitude is depicted in Fig. 7. It is evident that with this type of samples it is possible to achieve appreciable harmonic generation in the end sections applying linear excitation in the central section.

\section{Data acquisition system}

The main feature of the data acquisition system is that it is nonintrusive. The amplitude of the displacement is measured with a He-Ne laser vibrometer. The vibrometer measures frequencies of up to $1.5 \mathrm{MHz}$ and particle velocities between $10 \mathrm{microns} / \mathrm{s}$ and $10 \mathrm{~m} / \mathrm{s}$. The vibrometer is connected to a PC 386-computer to store and analyze the wave shapes. In the analysis FFT methods are applied. The temperature of the sample is monitored by an infrared thermometer. ${ }^{20}$

\section{RESULTS}

This paragraph presents the experimental results and their comparison with theoretical predictions.
To verify the assumption of one-dimensional behavior, measurements of the linear vibration velocity distribution along the different samples were carried out and compared with the corresponding results from the theoretical onedimensional approach. Figure 8 shows good agreement between the measurements and the theory and confirms the validity of the assumption.

The measurements were carried out for the different specimens at high excitation level. The results for uniformsection samples confirm the low harmonic generation that was theoretically predicted (Fig. 9).

For flexural vibrations, the frequency of the second resonance mode is never twice the fundamental mode. ${ }^{15}$ Therefore, the second resonance mode does neither coincide nor enhance the second harmonic, as it happens for extensional vibrations. To show the nonlinear behavior of the material more clearly, measurements were carried out driving the sample at a frequency $f_{2}$ such that twice this frequency coincides with the second resonance frequency of the bar. This way the second harmonic increased. The results obtained are shown in Fig. 10 for a uniform-section bar. Here, substantially higher harmonic generation can be observed. This situation is achieved by driving the samples with a finite amplitude (second harmonic generation at the driving point). Therefore, to achieve harmonic generation from linear excitation the above mentioned stepped samples were necessary.

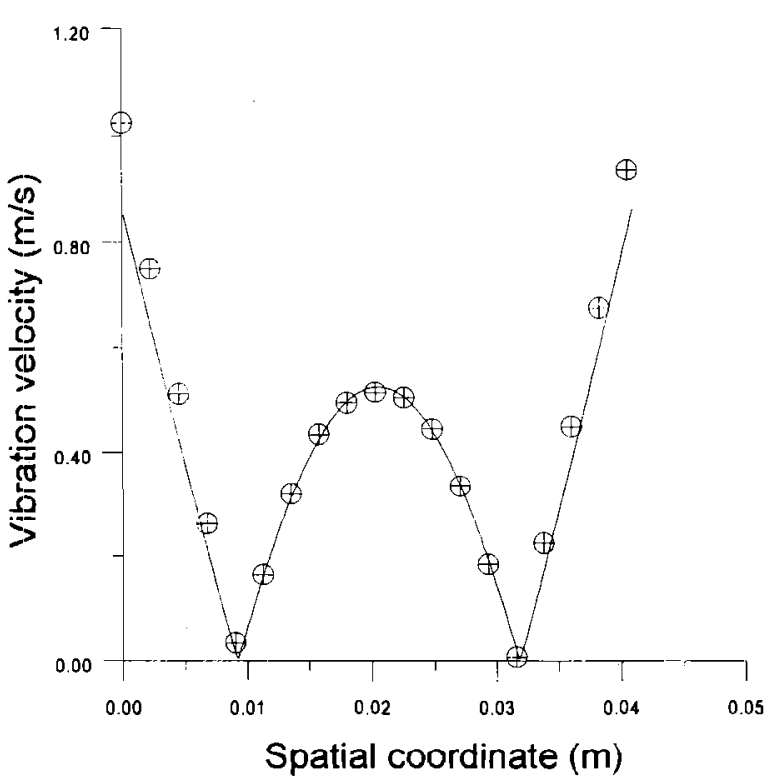

FIG. 8. Distribution of the vibration velocity amplitude of a uniform section bar vibrating at its first linear flexural mode. $\left(\dot{u}_{0}=0.5 \mathrm{~m} / \mathrm{s}\right)$ Experimental $(\oplus)$. Theoretical (— 


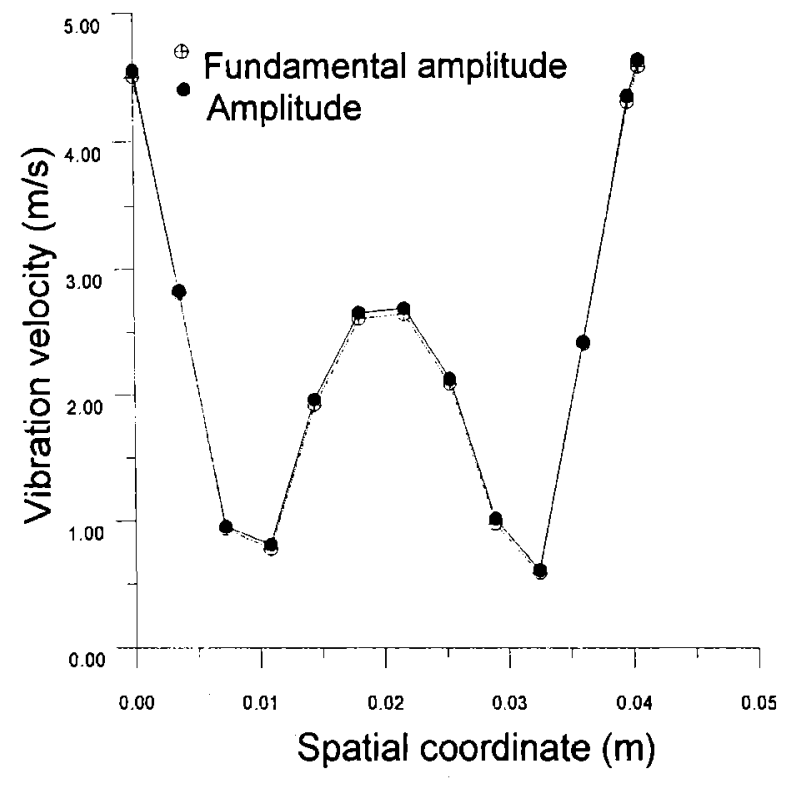

FIG. 9. Distribution of the experimental vibration velocity amplitude of a uniform section bar driven at its first flexural mode at high excitation level $\left(\dot{u}_{0}=2.6 \mathrm{~m} / \mathrm{s}\right)$. Total amplitude $(\bullet)$. Fundamental $(\oplus)$.

Stepped bars were used leading to effective strain increase in the external thinner zones located far from the excitation point (Fig. 7). The experimentally obtained result for a stepped bar $\left(l_{a} / l_{b}=1.7, h_{a} / h_{b}=3\right)$ driven at the frequency $f_{2}$ is shown in Fig. 11. In this case, a considerable harmonic generation is observed with linear excitation.

To compute the nonlinear, theoretical values for the stepped bar samples described above, the boundary conditions of Eq. (18) have to be applied to Eq. (15) together with the continuity conditions in every section change of the bar. From this, velocity, force, and momentum distributions are obtained. Figure 12 shows the calculated displacement dis-

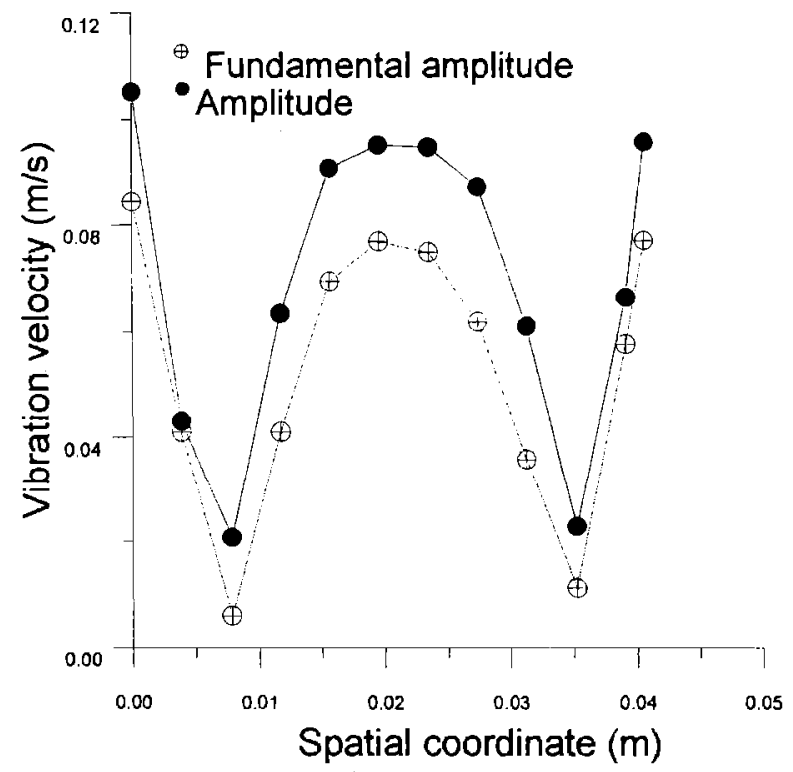

FIG. 10. Distribution of the experimental vibration velocity amplitude of a uniform section bar driven at the frequency $f_{2}$ (Excitation amplitude $\dot{u}_{0}=0.094 \mathrm{~m} / \mathrm{s}$ ). Total amplitude (ब). Fundamental $(\oplus)$.

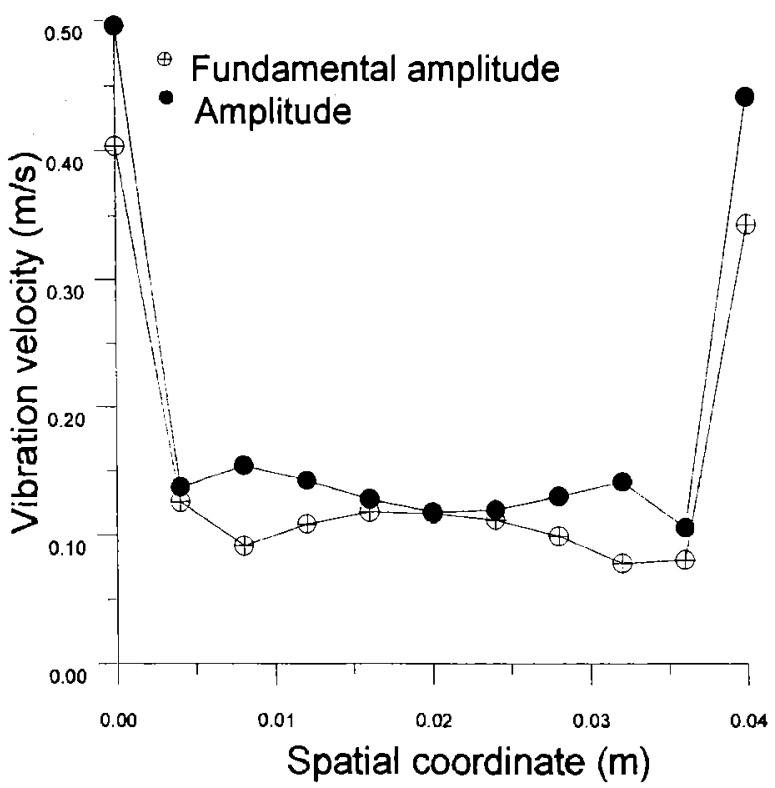

FIG. 11. Distribution of the experimental vibration velocity amplitude of a stepped bar driven at the frequency $f_{2}$ (Excitation amplitude $\dot{u}_{0}=0.12 \mathrm{~m} / \mathrm{s}$ ). Total amplitude $(\bullet)$. Fundamental $(\oplus) .\left(l_{a}=12.510^{-3} \mathrm{~m}, l_{b}=7.510^{-3} \mathrm{~m}\right.$, $\left.h_{a}=7.510^{-3} \mathrm{~m} \mathrm{y} h_{b}=2.510^{-3} \mathrm{~m}\right)$.

tribution along a stepped bar of $\mathrm{Ti} 6 \mathrm{Al} 4 \mathrm{~V}$ driven at its first flexural mode. It can be observed that for this geometry the nonlinear behavior is clearly stronger than for uniform bars. The distributions of the fundamental and second harmonic amplitude were also calculated for the stepped samples driven at the frequency $f_{2}$.

A comparison between the experimental and the calculated distribution of the fundamental and the second harmonic amplitude is shown in Fig. 13. The system was ex-

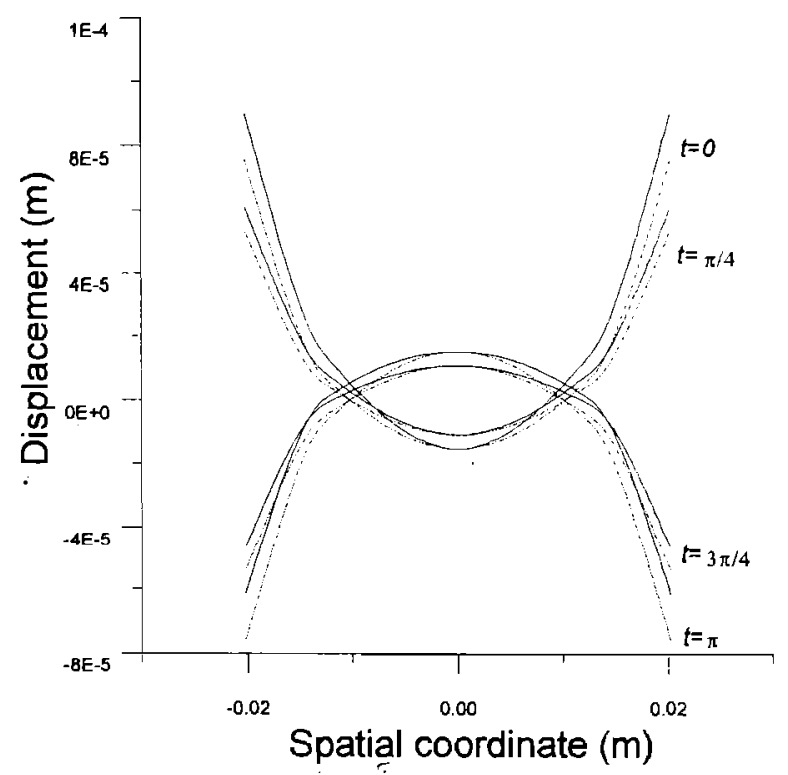

FIG. 12. Distribution of theoretical displacement in a stepped bar $\left(h_{a}=7.5\right.$ $\mathrm{mm}, h_{b}=2.5 \mathrm{~mm}, l_{a}=12.5 \mathrm{~mm}, l_{b}=8 \mathrm{~mm}$ ) vibrating at its first linear flexural mode at four different times within a period. Linear (-.............). Second-order approximation (-). (Excitation amplitude $\dot{u}_{0}=10 \mathrm{~m} / \mathrm{s}$. Nonlinearity parameter $\left.\beta_{E}=50\right)$. 


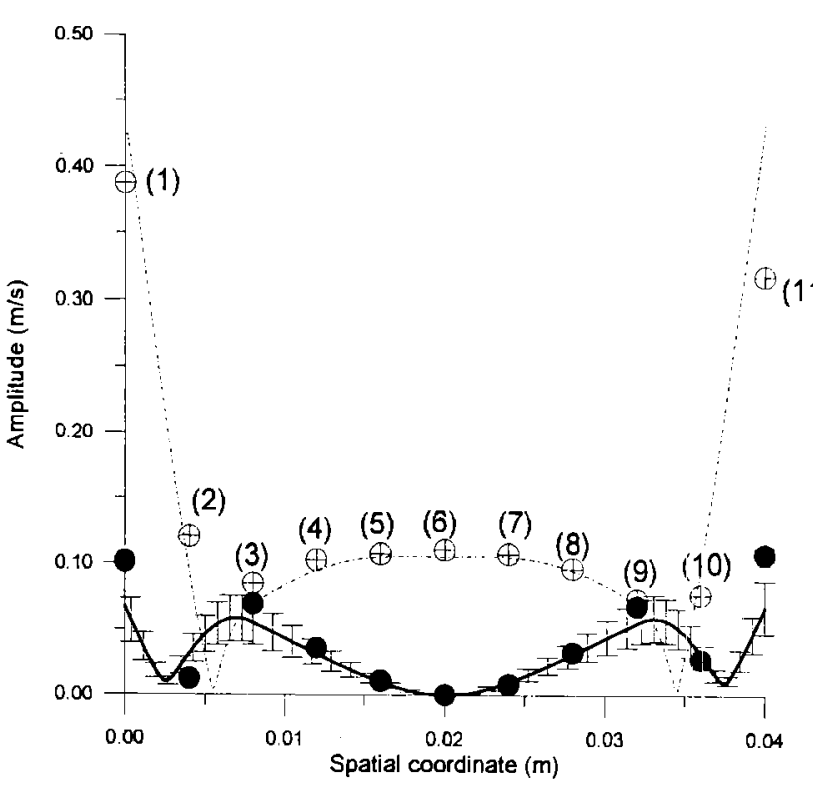

FIG. 13. Distribution of fundamental [Experimental $(\oplus)$ and theoretical $(-. . . . . . . . .)$.$] and second harmonic [Experimental (O) and theoretical$ (——)] amplitude of a stepped bar $\left(l_{a}=12.510^{-3} \mathrm{~m}, l_{b}=7.510^{-3} \mathrm{~m}\right.$, $\left.h_{a}=7.510^{-3} \mathrm{~m} y h_{b}=2.510^{-3} \mathrm{~m}\right)$ driven at the frequency $f_{2} \quad\left(\dot{u}_{0}=0.12\right.$ $\mathrm{m} / \mathrm{s} . \beta_{E}=28 \pm 15 \%$ ).

cited at the frequency $f_{2}$ to obtain significant nonlinear effects. All computed curves were calculated using the extensional nonlinearity parameter $\beta_{E}=28 \pm 15 \%$ previously obtained by the authors in a study of extensional, tinite amplitude standing waves in Ti $6 \mathrm{Al} 4 \mathrm{~V}$ rods. ${ }^{13}$ Figure 14 shows a comparison between experimental and calculated amplitudes of the fundamental as a function of excitation at two different points indicated in Fig. 13. Finally, a comparison between experimental and computed second harrnonic amplitudes is presented in Fig. 15 as a function of the fundamental at the same points as in Fig. 14. The good agreement between theory and experiment is evident from the figures.

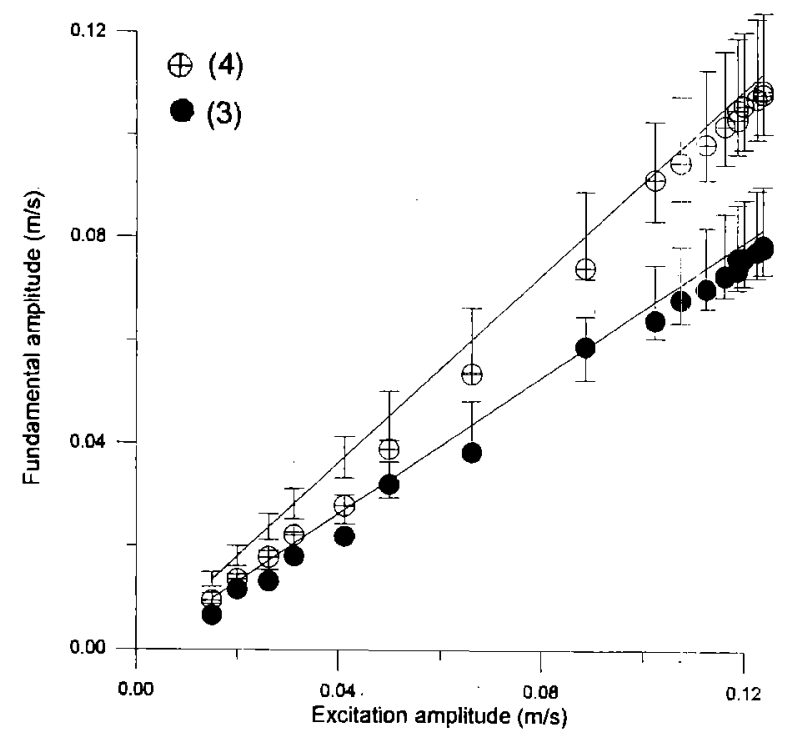

FIG. 14. Fundamental versus excitation amplitude at two different points (3 and 4 of Fig. 13) of the stepped bar. Experimental $(\oplus, 0)$ and theoretical $(\longrightarrow), \beta_{E}=28 \pm 15 \%$.

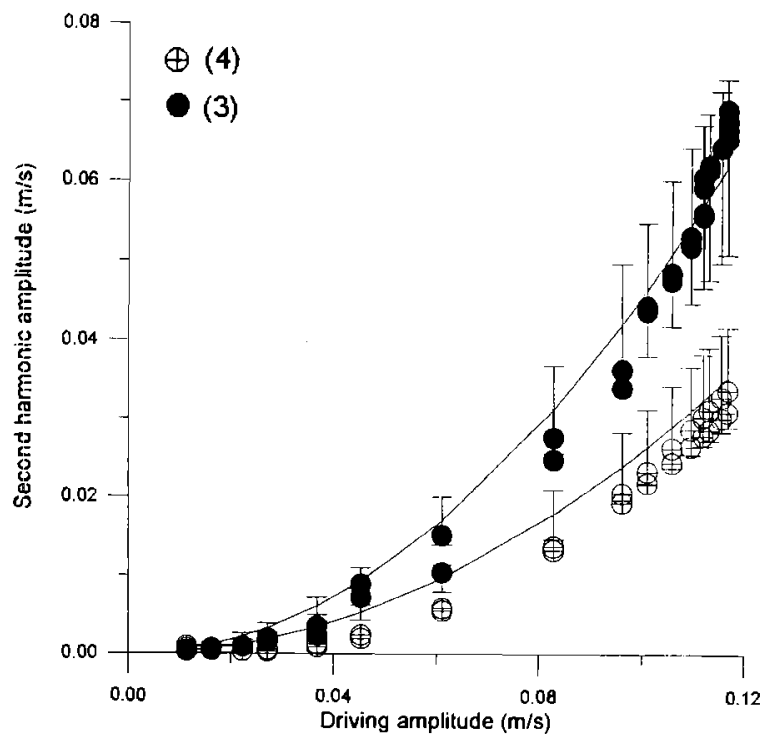

FIG. 15. Second harmonic versus excitation amplitude at two different points ( 3 and 4 of Fig. 13) of the stepped bar. Experimental $(\oplus, 0)$ and theoretical (—) $\beta_{E}=28 \pm 15 \%$.

As a consequence, the value of the nonlinearity parameter previously obtained is again verified.

\section{CONCLUSIONS}

Finite amplitude flexural waves in a titanium alloy $(\mathrm{Ti}$ $6 \mathrm{Al} 4 \mathrm{~V}$ ) were studied theoretically and experimentally. The theoretical approach consisted of a one-dimensional model based on nonlinear elasticity theory. Superposition of two finite amplitude waves, propagating in positive and negative spatial direction, was assumed. The model predicts minor nonlinear behavior for this type of waves. This result supports the applicability of the flexural vibrations for high power sonic and ultrasonic transducers.

To achieve higher strain amplitudes, the experimental work was carried out with stepped prismatic bars. The measurements showed good agreement with theoretical predictions. This agreement verifies the value for the compressional nonlinearity parameter $\beta_{E}$ for the Ti $6 \mathrm{Al} 4 \mathrm{~V}$ material which was obtained in an earlier paper by the authors. ${ }^{13}$

\section{ACKNOWLEDGMENTS}

This research was supported by the Plan Nacional de Tecnologías Avanzadas de la Producción (research project TAP 93-230). The authors thank Dr. F. Montoya-Vitini and Mr. P. T. Sánz-Sánchez for the design and construction of the electronic excitation system and Dr. T. Hoffmann for revision.

'F. D. Murnaghan, Finite Deformation of an Elastic Solid (Wiley, New York, 1951).

${ }^{2}$ R. N. Thurston, in Physical Acoustics, edited by W. P. Mason (Academic, New York, 1964), 1 A, pp. 1-110.

${ }^{3}$ R. N. Thurston and M. J. Shaphiro, J. Acoust. Soc. Am. 41, 1112-1/25 (1967).

${ }^{4}$ M. A. Breazeale and J. Philip, in Physical Acoustics, edited by W. P. Mason and R. N. Thurston (Academic, Orlando, 1984), Vol. XVII, pp. I -60 . 
${ }^{5}$ J. A. Gallego-Juárez, "High-power ultrasonic transducers for use in gases and interphases," in Power Sonic and Ultrasonic Transducers Design (Springer-Verlag, Berlin, 1988), Chap. 10, pp. 175-184.

${ }^{6}$ P. A. Johnson, G. D. Meegan, K. R. Mccall, T. J. Shankland, R. A. Guyer, and B. P. Bonner, in Advances in Nonlinear Acoustics, edited by H. Hobaek (World Scientific, Singapore, 1993), pp. 525-530.

${ }^{7} \mathrm{~J}$. K. Na and M. A. Breazeale, in Frontiers in Nonlinear Acoustics, edited by M. F. Hamilton and P. T. Blackstock (Elsevier, London, 1990), pp. 571-576.

${ }^{8}$ K. R. McCall, in Advances in Nonlinear Acoustics, edited by H. Hobaek (World Scientific, Singapore, 1993), pp. 519-524.

${ }^{9}$ B.-T. Chu, J. Mech. Phys. Solids 12, 45-57 (1964)

${ }^{10}$ A. L. Van Buren, J. Sound Vib. 42, 273-280 (1975).

${ }^{11}$ M. Dah-You, Chin. J. Acoust. 9(3), 193-204 (1990)

${ }^{12}$ P. A. Johnson, P. Rasolofosaon, and B. Zinszner, in Advances in Nonlinear Acoustics, edited by H. Hobaek (World Scientific, Singapore, 1993), pp. 531-536.
${ }^{13}$ C. Campos-Pozuelo and J. A. Gallego-Juárez, J. Acoust. Soc. Am. 97, 875-881 (1995).

$14 \mathrm{~J} . \mathrm{J}$. Gagnepain, in Ultrasonic Methods in Evaluation of Inhomageneous Materials, edited by Alippi and W. G. Mayer (Nijhoff, Dordrecht, 1987), pp. 243-262.

${ }^{15}$ P. M. Morse and K. U. Ingard, Theoretical Acoustics (McGraw-Hill, New York, 1986).

${ }^{16}$ M. A. Breazeale, in Advances in Nonlinear Acoustics, edited by $\mathrm{H}$. Hobaek (World Scientific, Singapore, 1993), pp. 451-456.

${ }^{17}$ J. A. Gallego-Juárez, G. Rodriguez-Corral, J. L. San Emeterio, and F. Montoya-Vitini, European Patent No. EP 450030 (1991).

${ }^{18}$ C. Campos-Pozuelo, J. A. Gallego-Juárez, and P. T. Sanz-Sánchez, Ultras. Int. 93 Conf. Proceedings (Butterworth-Heinemann, Oxford, 1993), pp. 571-574.

${ }^{19}$ J. A. Gallego Juárez, J. Sound Vib. 26 (3), (1973).

${ }^{20}$ R. B. Minogna, R. E. Green, Jr., J. C. Duke, Jr., E. G. Henneke, and K. L. Reifsnider, Ultrasonics 19, 159-163 (1981). 